\title{
Traumatic Arteriovenous Malformation of Cheek: A Case Report and Review of Literature
}

\author{
Vadisha Srinivas Bhat, Rajeshwary Aroor, B Satheesh Kumar Bhandary, Shama Shetty
}

\section{ABSTRACT}

Arteriovenous malformations (AVM) are congenital vascular anomalies but are usually first noticed in childhood or adulthood. Head and neck is the most common location for AVM. Extracranial lesions are rare compared to intracranial lesions. The rapid enlargement of the malformation leading to symptoms is usually triggered by trauma or hormonal changes of puberty or pregnancy. Traumatic AVM of the head and neck are very rare. Here we report a case of AVM of cheek in an adult woman developed following a dental treatment. The diagnosis was confirmed by imaging and was treated surgically after angiography and embolization.

Keywords: Arteriovenous malformation, Cheek, Dental procedure, Angiography, Embolization.

\section{Source of support: Nil}

Conflict of interest: None declared

\section{INTRODUCTION}

Arteriovenous malformations are congenital vascular anomalies but are usually first noticed several years after birth. The rapid enlargement of the malformation is usually triggered by trauma or hormonal changes during puberty or pregnancy. Enlargement of the lesion is due to the changes in pressure and flow, dilatation of vascular channels, shunting and collateral proliferation, rather than cellular proliferation. ${ }^{1}$ The delay is diagnosis is either due to the dormant lesion expanding later, or due to the misdiagnosis of the malformation as hemangioma, especially in small vessel rich AVM, where even imaging may not differentiate the lesion from hemangioma. ${ }^{2}$ Head and neck is the most common location for arteriovenous malformations. Extracranial lesions are rare compared to intracranial lesions. ${ }^{3}$ Clinical diagnosis of AVM can be confirmed by imaging- Doppler ultrasonography, CT scan with contrast and magnetic resonance imaging (MRI) will give a definite diagnosis. ${ }^{4}$ Angiogram shows dilated tortuous arteries, arteriovenous shunting and dilated draining veins, but this is useful only if embolization is planned. Surgical excision following selective embolization is the treatment of choice. ${ }^{1}$ Very few cases of traumatic AVM are reported in the literature. We report a case of AVM of cheek in an adult woman, following dental procedure.

\section{CASE REPORT}

A 31-year-old woman presented with swelling on right cheek of 6 months duration, which was insidious in onset and gradually increasing in size. She had a history of undergoing dental treatment on the right upper premolar tooth 1 month before the onset of swelling. There was no history of any other trauma to the face. There were no symptoms of nasal disease. On examination, her general health state was good. There was a swelling measuring $3 \times 2 \mathrm{~cm}$ on the right cheek near the nasolabial grove which was soft, compressible and nontender. The swelling was pulsatile (Figs 1 and 2). Nasal cavity and paranasal sinuses were within normal limits. Oral cavity was normal. Ultrasonography showed a hypoechoic area with central necrosis measuring $15 \times 10 \mathrm{~mm}$ in right premaxillary region. On color Doppler, extensive vascularity noted consisting of arterial and venous channels. Adequate vascular channels suggested feeders from branch of maxillary artery. The sonological features were suggestive of AV malformation. Contrast enhanced CT scan of paranasal sinus showed intensely enhancing mass in right premaxillary soft tissue, measuring $2.2 \times 2.0 \times 2.0 \mathrm{~cm}$, with prominent vessels surrounding it, suggesting vascular malformation (Figs 3 to 5). MRI of the maxillofacial region showed a well defined rounded lesion in right premaxillary and nasolabial region measuring $3.5 \times 2 \mathrm{~cm}$ in size. Multiple enlarged flow voids seen within it suggesting high vascularity of malformation (Fig. 6). Imaging studies were diagnostic of an arteriovenous malformation.

Right external carotid angiogram showed moderate size (3-4 cm), compact, moderate flow right premaxillary and nasolabial arteriovenous malformation supplied by hypertrophied angular and anterior jugular branch of facial artery, infraorbital and buccal branches of distal internal maxillary, jugular branches of transverse facial artery. Venous drainage was inferiorly into facial vein and superiorly through angular vein into contralateral angular vein and also into right superior ophthalmic vein (Fig. 7). Selective embolization of the vessels was done with gel foam, attaining more than $50 \%$ reduction in vascularity. Complete excision of the mass with ligation of the vessels, was done by lateral rhinotomy incision, elevating the facial skin flap off the mass, under general anesthesia, the following day of embolization (Fig. 8). There was no 


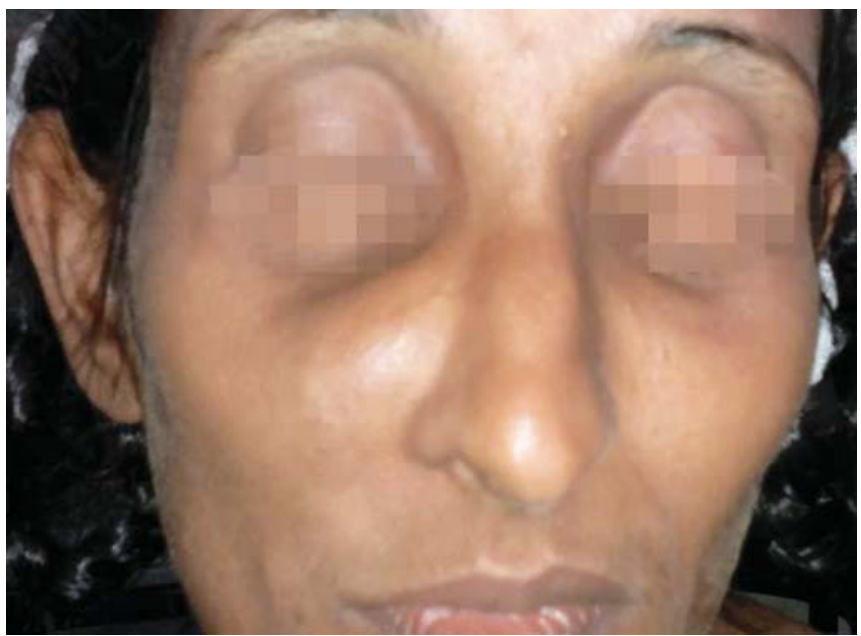

Fig. 1: Clinical photograph (anterior view)

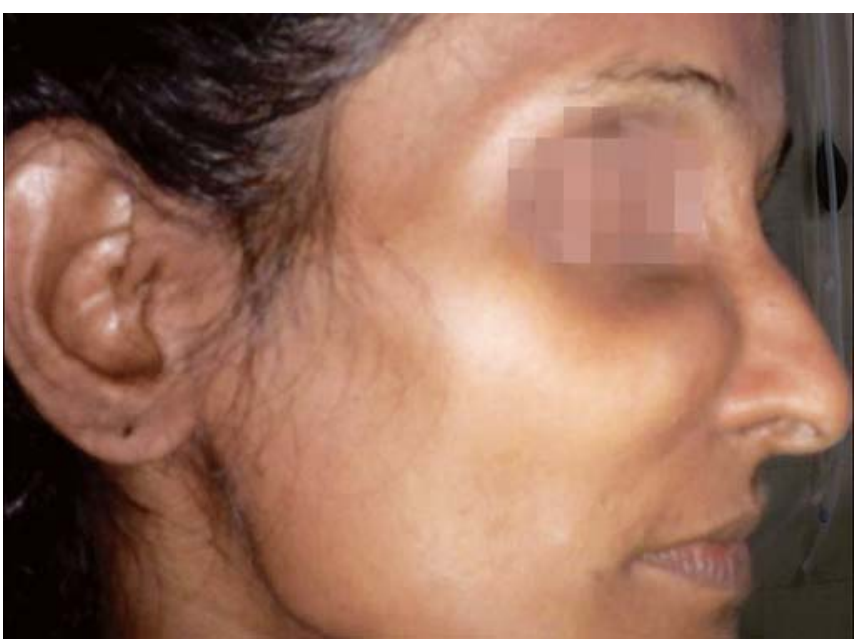

Fig. 2: Clinical photograph (right lateral view)

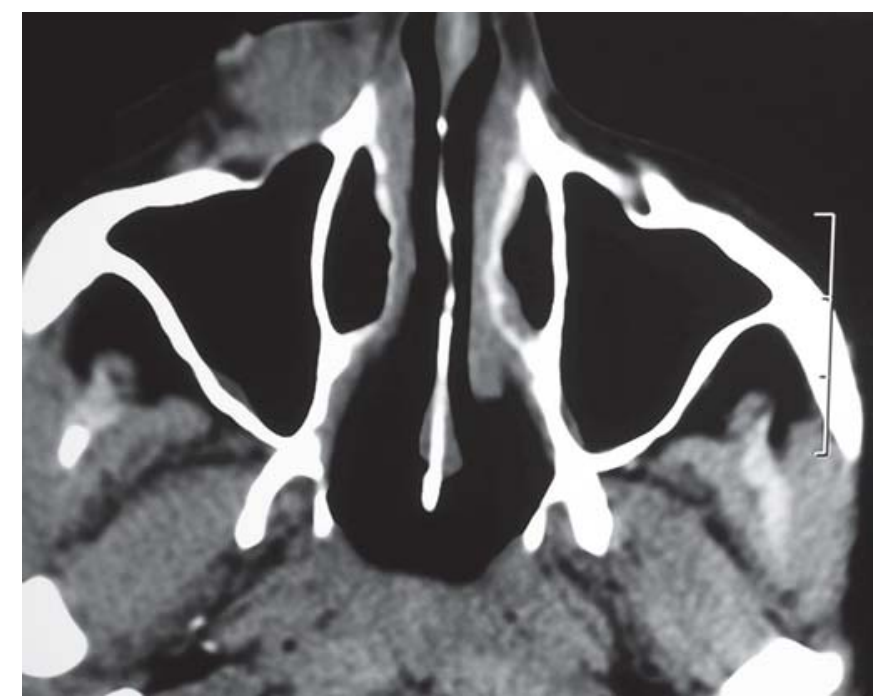

Fig. 3: CT scan paranasal sinus (axial view plain)

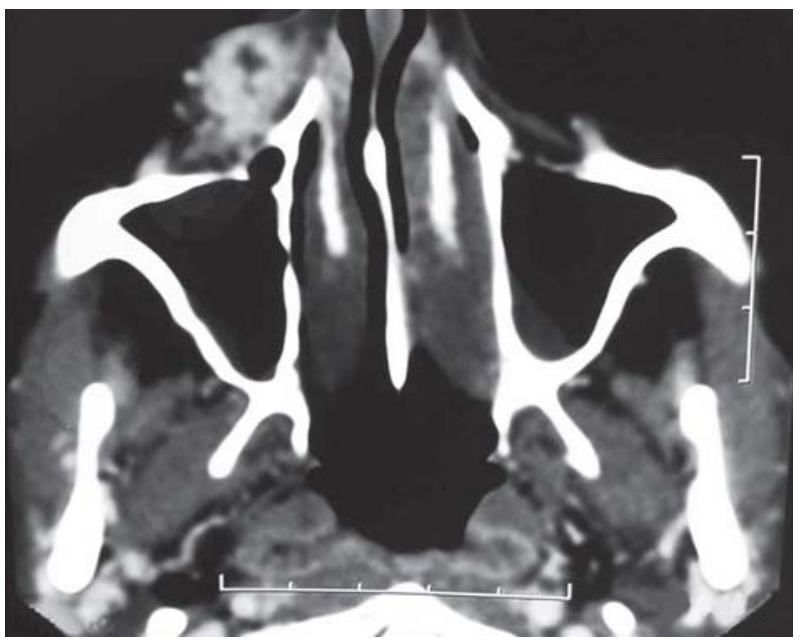

Fig. 4: CT scan paranasal sinus-axial view post-contrast showing intensely enhancing region in right premaxillary soft tissue

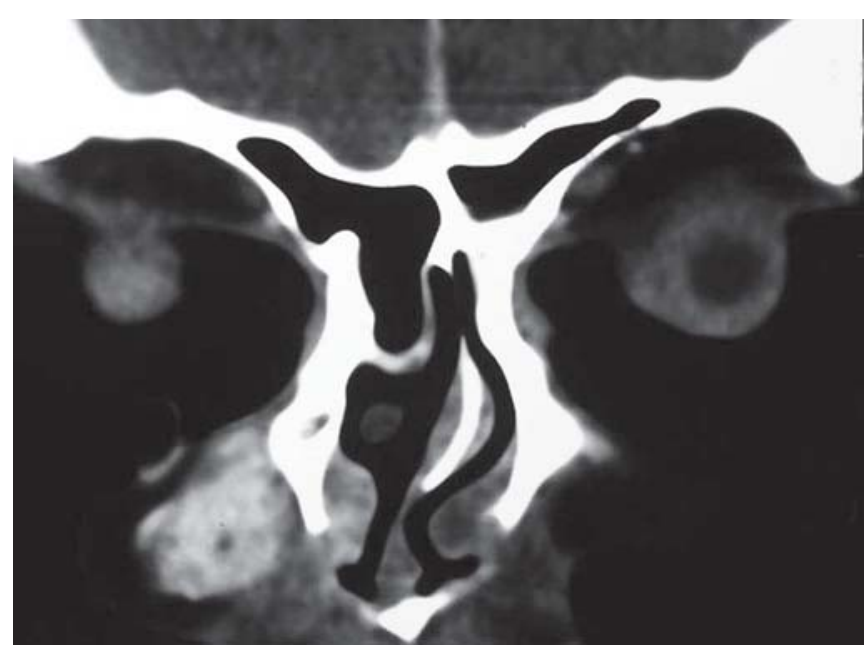

Fig. 5: CT scan of paranasal sinus (coronal view post-contrast)

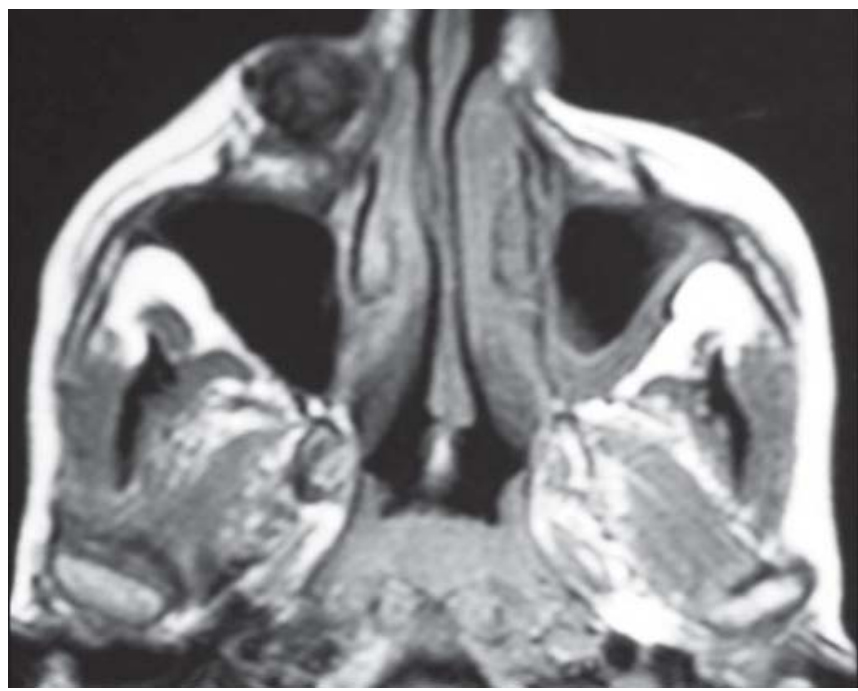

Fig. 6: MRI showing a well-defined rounded lesion in right premaxillary and nasolabial region 

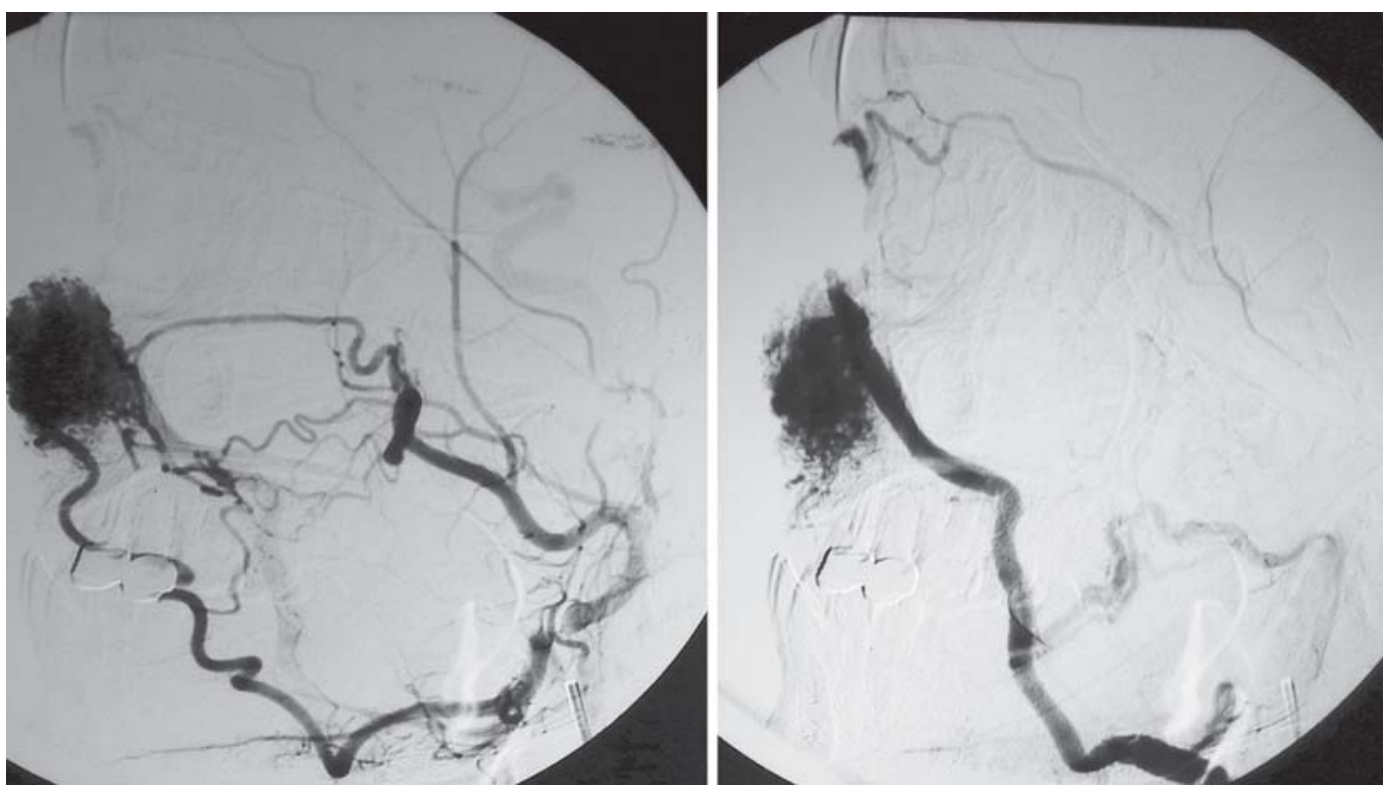

Fig. 7: Right external carotid angiogram showing right premaxillary and nasolabial arteriovenous malformation

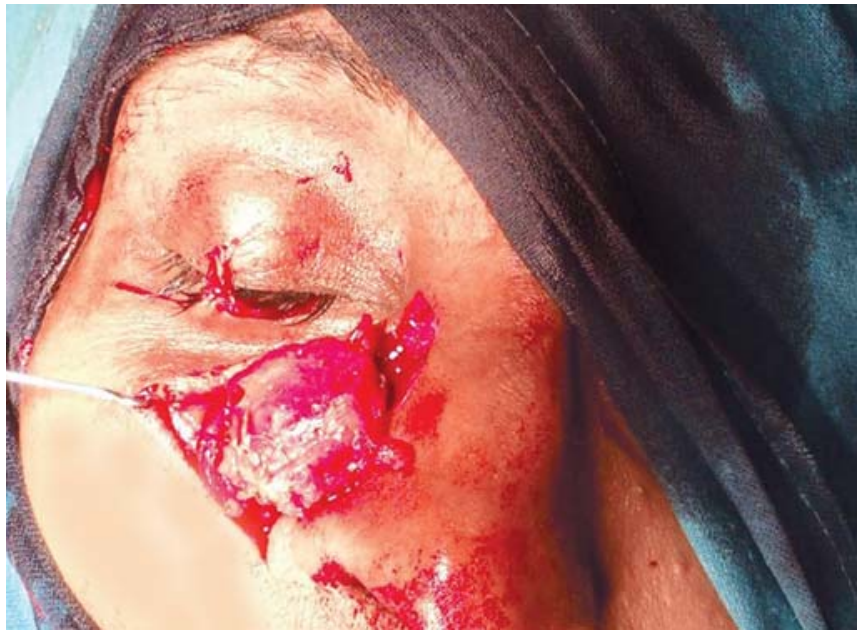

Fig. 8: Excision of mass by lateral rhinotomy incision 3.

significant bleeding during surgery. Patient withstood the procedure well. Postoperative period was uneventful. She was discharged from the hospital 6 days after surgery, after suture removal.

Histopathological examination showed large dilated vascular channels with thrombi. Surrounding these large vascular channels is proliferation of capillaries in lobules with stasis of blood. Histological features were confirmatory of arteriovenous malformation (Figs 9 and 10). As the patient hails from a distance place, she could not visit us after surgery; however she is in touch with us on telephone. She is not having any complaints pertaining to the disease 1 year after surgery.

\section{DISCUSSION}

Vascular malformations are a form of vascular anomaly caused by abnormal development of vascular elements

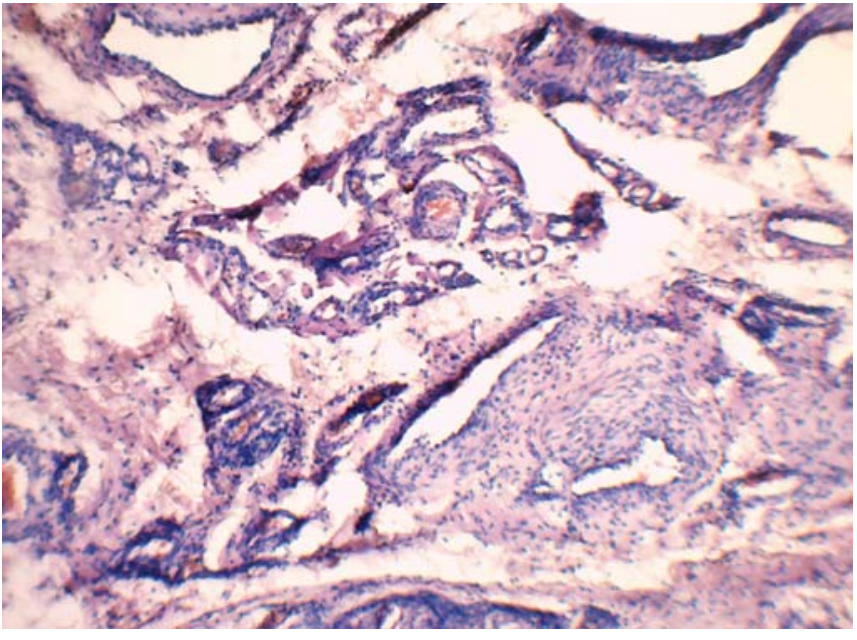

Fig. 9: H\&E stain, low power showing vascular channels of various size

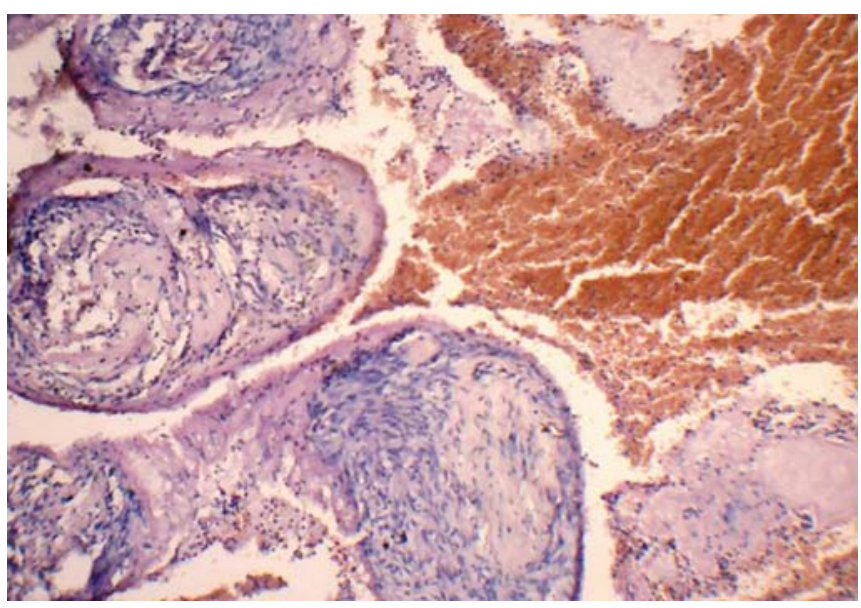

Fig. 10: H\&E high power showing vascular channels with thrombus

during embryogenesis and in fetal life. ${ }^{4}$ These vascular malformations do not produce increased endothelial cell 
turnover as seen in vascular tumors like hemangioma. ${ }^{4}$ These malformations can be of a single vessel form or in combination of arterial, venous, capillary or lymphatic malformation. Vascular malformations can be a slow flow malformation like capillary, lymphatic or venous; or it can be a fast flow like arterial or arteriovenous malformation. ${ }^{3}$

Arteriovenous malformations (AVM) are fast-flow vascular malformations, consisting anomalous capillary beds between the arterial and venous system, thus causing shunting of blood. AVMs are the most aggressive form of vascular malformations, which can lead to significant deformity and functional impairment. ${ }^{2}$ They are usually noted at birth, but are commonly misdiagnosed as other vascular anomalies leading to delay in diagnosis. ${ }^{3}$ Fast flow becomes evident during childhood and puberty. ${ }^{4}$ Kohout MP et al in their study of AVM of head and neck, found in $59 \%$ of cases, AVM was present at birth. Ten percent noted in childhood, $10 \%$ in adolescent and $21 \%$ in adulthood. ${ }^{5}$ AVM grow synchronously with the growth of the child. Puberty and trauma are found to have triggered the rapid growth of the lesion leading to manifestation of the symptoms. ${ }^{3}$ Enlargement is the result of changes in the pressure and flow within the malformation, ectasia, shunting and collateral proliferation, rather than cellular proliferation. ${ }^{1}$ Though the pathogenesis of AVM is not clear, there are theories like defects in TGF-beta signaling and genetic two hit hypothesis causing the AV malformation. ${ }^{6,7}$ Also, to explain the expansion seen during puberty, Progesterone receptors are isolated in $\mathrm{AVM}^{8}{ }^{8}$ The patient in this report is an adult woman, presented with the swelling at the age of 30 years, following a dental procedure adjacent to the site of lesion. Though not definite, the onset of the symptom may be attributed to the trauma caused during the dental procedure. Traumatic AV malformations of facial region are very rare. In literature approximately 30 cases are angiographically documented traumatic AVM of the face are reported. ${ }^{9}$ Holt GR et al reported that the trauma leading to the AVM may be penetrating, blunt, postsurgical or inflammatory. ${ }^{9}$ This statement holds good in our case, as the impact of trauma in this case is trivial.

The presenting symptoms of facial AVM include swelling over the face, which is noticed incidentally. Further symptoms depend on the clinical staging introduced by Schobinger in $1990 .^{10}$

Stage I (quiescence): Pink-bluish stain, warmth and arteriovenous shunting demonstrated by Doppler.

Stage II (expansion): Stage I lesion with enlargement, pulsations, thrill, bruit and tense/tortuous veins.

Stage III (destruction): Stage II lesion with dystrophic skin changes, ulceration, tissue necrosis, bleeding or persisting pain.
Stage IV (decompensation): Features of cardiac failure caused by an extensive AVM causing increased cardiac output.

The AVM can infiltrate causing local tissue destruction and often lead to life-threatening hemorrhage. Our patient presented with a swelling over the cheek which was pulsatile, and the AVM was demonstrated by imaging. Hence she was considered in stage II.

History and clinical examination can distinguish vascular tumors and vascular malformations in over $90 \%$ of cases. ${ }^{4}$ However types of malformation can be confirmed with ultrasound with color Doppler examination. CT scan with iodinated contrast identifies AVMs as a highly enhancing lesion and can demonstrate soft tissue involvement, as well as dilated feeding and draining vessels. ${ }^{11}$ Magnetic resonance imaging of an AVM demonstrates a collection of vascular flow voids corresponding to fast-flow vessels and it can be considered the investigation of choice due to its ability to delineate the extent of the lesion, provide multiplane images and difference between high and low flow lesions. MRI is superior to magnetic resonance angiography (MRA) and conventional cerebral angiography CCA in showing the nidus and the involved brain structures, in cases of AVM of brain. ${ }^{12}$ Even on MRI, small vessel rich AVMs, often appear as a well-circumscribed tumor like vascular mass, mimicking hemangioma. Biopsy may be considered for histological diagnosis when the imaging findings conflict clinical findings. ${ }^{2}$ Angiography demonstrates variably dilated or tortuous feeding arteries, arterial venous shunting occasionally with visualization of discrete fistulae, and dilated draining veins. ${ }^{5,11}$

AVMs are the most difficult vascular anomaly to manage due to the replacement of normal tissue by the disease vessels and the high rate of recurrence. ${ }^{3}$ Combined embolization and surgical resection is most successful for well-localized AVMs. Both the AVM nidus and involved skin must be excised widely, although if the overlying skin appears normal, it can be saved. Excision is performed 24 to 48 hours after embolization. Absolute ethanol, polyvinyl alcohol and gel foam can be used for embolization. ${ }^{3}$ Ligation or proximal embolization of feeding vessels alone should never be done, which can lead to recruitment of new vessels from the adjacent area to supply the nidus. ${ }^{4}$ Embolization helps in reducing the blood loss and delineating the mass during dissection. Regular followup with Doppler sonography is suggested as the disease has a high recurrence rate. In our patient, CT scan, Doppler ultrasonography, MRI and carotid angiography was suggestive of right nasolabial arteriovenous malformation with feeder from right maxillary artery. She was treated by selective embolization followed by complete resection of 
the mass. Skin was not excised as it was grossly normal and was not firmly adherent to the mass. Follow-up period of 1 year is uneventful; there is no evidence of recurrence.

Sclerotherapy of AVM has a limited role. Various sclerosing agents that are used include sodium morrhuate, boiling water, nitrogen mustard. It can be used to control the symptoms in addition to embolization, in a case where complete excision of the mass is not possible. ${ }^{4}$

\section{CONCLUSION}

Early diagnosis and treatment of AVMs is important, as the natural history of AVMs is progressive expansion and associated morbidity. The likelihood of cure may be improved with treatment of AVMs at an earlier stage of development. Detailed investigations including Doppler ultrasound, MRI and CT scan are required to develop an appropriate treatment plan. Appropriate management is best achieved via a multi-disciplinary approach. Combined embolization and surgical resection is most successful for well-localized AVMs. However, these patients must still be followed for years with regular physical examination, ultrasonography and/or MRI.

\section{REFERENCES}

1. Bhandari PS, Sadhotra LP, Bhargava P, Bath AS, Mukherjee MK, Maurya S. Management strategy for facial arteriovenous malformations. Indian J Plast Surg 2008 Jul-Dec;41(2):183-189.

2. Jakubowski LA, Chun RH, Drolet BA, Jensen JN, North PE. Misdiagnosis as infantile hemangioma: early presentation of small vessel-rich AVM. Int J Pediatrr Otorhinolaryngol Extra (2013), http:///dx.doi.org/10.1016/j.pedex.2013.04.002.

3. Gresham TR, Adva BF. Hemangiomas and vascular malformations: current theory and management. International Journal of Pediatrics 2012, Article ID 645-678.

4. Marler JJ, Mulliken JB. Current management of hemangiomas and vascular malformations. Clin Plastic Surg 2005;32:99-116.

5. Kohout MP, Hansen M, Prebaz JJ, Mulliken JB. Arterio venous malformation of the head and neck: natural history and management. Plast Reconstr Surg 1998;102:643-654.
6. Corti P, Young S, Chen CY, et al. Interaction between alk1 and blood flow in the development of arteriovenous malformations. Development 2011;138(8):1573-1582.

7. Kim H, Su H, Weinsheimer S, Pawlikowska L, Young WL. Brain arteriovenous malformation pathogenesis: a response-toinjury paradigm. Acta Neurochirurgica, Supplementum 2011;111:83-92.

8. Dukya LJ, Fan CY, Coviello-Malle JM, Buckmiller L, Suen JY. Progesterone receptors identified in vascular malformations of the head and neck. Otolaryngology-Head and Neck Surgery 2009;141(4):491-495.

9. Holt GR, Holt JE, Cortez EA, Thornton WR, Young WC. Traumatic facial arteriovenous malformations laryngoscope. 1980 Dec;90(12):2011-2020.

10. Mulliken JB. Vascular anomalies. In: Aston SJ, Beasley RW, Thorne CHM, editors. Grabb and Smith's plastic surgery. Philadelphia: Lippincott-Raven 1997. P. 191-204.

11. Lee BB, Baumgartner I, Berlien HP, Bianchini G, Burrows P, Do YS, et al. Consensus Document of the International Union of Angiology (IUA)-2013 Current concepts on the management of arterio-venous malformations. Int Angiol 2013;32(1):9-36.

12. Nussel F, Wegmuller H, Huber P. Comparison of magnetic resonance angiography, magnetic resonance imaging and conventional angiography in cerebral arteriovenous malformation. Neuroradiology 1991;33:56-61.

\section{ABOUT THE AUTHORS}

\section{Vadisha Srinivas Bhat (Corresponding Author)}

Associate Professor, Department of Otorhinolaryngology, KS Hegde Medical Academy, Mangalore, Karnataka, India, Phone: 08242204491 e-mail: bvadish@yahoo.co.in

\section{Rajeshwary Aroor}

Professor, Department of Otorhinolaryngology, KS Hegde Medical Academy, Mangalore, Karnataka, India

\section{B Satheesh Kumar Bhandary}

Professor, Department of Otorhinolaryngology, KS Hegde Medical Academy, Mangalore, Karnataka, India

\section{Shama Shetty}

Resident, Department of Otorhinolaryngology, KS Hegde Medical Academy, Mangalore, Karnataka, India 